\title{
Jenseits der Gründungsmythen - Kiel und die Historische Geographie
}

\author{
J.-E. Steinkrüger \\ Geographisches Institut, Bonn, Germany \\ Correspondence to: J.-E. Steinkrüger (steinkrueger@giub.uni-bonn.de)
}

Received: 18 August 2015 - Accepted: 2 September 2015 - Published: 21 September 2015

Es scheint rein koinzident, dass 1969, genau im Jahr des zum Mythos gewordenen Kieler Geographentages, das erste und bis 2011 letzte Lehrbuch für Historische Geographie im deutschsprachigen Raum erschien, das die Historische Geographie explizit als Teil der Geographie auffasste (Jäger, 1969; Schenk, 2011). Auch ist es vermutlich zufällig oder eher Ausdruck der Persönlichkeit Helmut Jägers, des Verfassers jenes Bandes in der Westermann-Reihe Das Geographische Seminar, dass er sich in jener berühmt gewordenen Sitzungen über „Der Geograph - Ausbildung und Beruf" zu Wort meldete. Er berichtete darin, dass die von den Studierenden eingeforderte Entkoppelung von Physischer Geographie und Humangeographie sowohl an der Universität Würzburg durch mögliche Schwerpunktsetzungen in Diplom- und Promotionsstudium als auch durch Unterscheidung der Gutachter nach Human- und Physischer Geographie in der Deutschen Forschungsgemeinschaft voranschreite (Meckelein und Borcherdt, 1970). Dem historischen Ereignis Kiel fügen beide Beobachtungen lediglich eine Randnotiz bei. Und dennoch ist der Mythos Kiel auch in die Historische Geographie eingeschrieben. Als Aufstieg und Siegeszug moderner Wissenschaftlichkeit und anwendungsorientierter Sozialgeographie zelebriert, war Kiel aus Perspektive der Historischen Geographie Symbol von Marginalisierung, Exklusion und steigendem Legitimationszwang und dies zum Teil bis in die jüngste Vergangenheit. Historische Geographie wurde zu dem Anderen, von dem sich eine „neue“ Geographie lossagte. Denn im geographischen Fachdiskurs nach Kiel wurde der Begriff „Historische Geographie" synonym für jenes ancien regime stark morphogenetischen ausgerichteter Kultur(landschafts)geographie angesehen, welche in vielerlei Hinsicht zu Recht als „unwissenschaftlich" nicht nur im Sinne kritisch-rationalistischer Einheitswissenschaftsideologien abgelehnt wurde. Auch und vielleicht noch bedeutender wirkte historisch-geographische
Forschung wenig zukunftsverändernd und deshalb wenig problem- und gesellschaftsrelevant; eine Kritik, die eher szientistisch orientierte, „liberale Reformer“ mit „linksrevolutionären" Anhängern der Kritischen Theorie einte. Mittelfristig hatte dies auch institutionelle Folgen: „Dieser Prozess war für den historischen Ansatz in der Geographie durchaus existentiell, denn das bedeutete die Loslösung der allgemeinen Siedlungs- und Kulturgeographie vom genetischen Forschungs- und Erklärungsansatz, was sich in der Umwidmung von Professuren der Kulturgeographie hin zu Forschungsfeldern niederschlug, die als zukunftsfähiger angesehen wurden“" (Schenk, 2011:5).

Besonders deutlich wird der Bruch nach Kiel in Gerhard Hards Die Geographie. Eine wissenschaftstheoretische Einführung. Darin begrüßt er das Aussterben des landschaftsgenetischen Ansatzes in der deutschen Geographie und befürwortet dessen Rückzug in die Historische Geographie. „wo sie, disziplinhistorisch gesehen, auch herstammt" (Hard, 1973:157). Dabei räumt er zumindest in einer Endnote selbst ein (Hard, 1973:273-274), dass die Historische Geographie als eigene Fachdisziplin zu dieser Zeit nicht existierte und zudem auch nicht notwendig mit Kulturlandschaftsforschung gleichzusetzen sei. Solche Aussagen setzten allerdings diejenigen, die einen historischen Zugang pflegten, in das Außen einer neuen Geographie, die zudem in den 1970ern mögliche gemeinsame Wurzeln und Kontakte auszumerzen versuchte. Rückblickend beschreibt Hans-Jürgen Nitz (1992:212) die Situation etwas überspitzend: „In dieser Periode wurden derartige Themen [gemeint sind Themen der historischgenetischen Kulturgeographie] für eine Vorstellung auf dem Deutschen Geographentag nicht mehr genommen. Viele junge Geographen, die ihre Karriere als historische Geographen begonnen hatten, wandten sich neuen Themen und Richtungen zu.“ 
Wie rapide sich Geographie und Historische Geographie trennten, zeigen auch zwei Innenansichten der Historischen Geographie, zwischen denen nur sechs Jahre lagen ${ }^{1}$ : Im bereits genannten Lehrbuch verortet Helmut Jäger (1969:11) die Historische Geographie deutlich in der Geographie als „Geographie in ihrer ganzen Fülle und Breite mit Einschluß von Zweigen der Allgemeinen Geographie und der Länderkunde; im Gegensatz zur primär gegenwartsbezogenen Geographie geht es ihr jedoch in erster Linie um die Erforschung der geographischen Verhältnisse der Vergangenheit“.

Im Gegensatz dazu definiert Klaus Fehn (1975:49) kurze Zeit später „Historische Geographie“ als außerhalb der Geographie „selbständige Wissenschaft, die sowohl für die Gegenwartsgeographie als auch für die Geschichtswissenschaften eine wichtige Hilfswissenschaft darstellt. Falls der Geographie nicht nur die Aufgabe zugewiesen wird, die Verhältnisse der Gegenwart zu erforschen, kann die Historische Geographie auch als Teil der Gesamtgeographie bezeichnet werden. Die Historische Geographie ist dementsprechend Geographie im umfassenden Sinne, die sich im Gegensatz zur Gegenwartsgeographie nur nicht mit der Gegenwart, sondern mit der Vergangenheit beschäftigt“.

Diese „Wenn-dann-Bedingung“ (Schenk, 2011:7) und Selbstbeschreibung als Hilfswissenschaft ist zum Teil bis heute kennzeichnend für das Verhältnis von Geographie und Historischer Geographie und dies, obwohl die Historische Geographie institutionell heute faktisch Subdisziplin der Geographie geworden ist, da die letzten verbliebenen Arbeitsgruppen in Bonn und Bamberg, die explizit den Namen „Historische Geographie“ tragen, beide in geographischen Instituten beheimatet sind. Wenn eine geographische Fragestellung sich auf eine Vergangenheit bezieht - sei es in Form der Rekonstruktion einer Vergangenheit, eines diachronen Vergleichs oder der Genese eines gegenwärtigen Phänomens (Nitz, 1992:215-216) - und wir dies noch als „Geographie“ bezeichnen, dann können wir damit Historische Geographie „als einen Betrachtungsansatz der Geographie [...] verstehen, der sich auf alle Teildisziplinen der Geographie beziehen kann" (Schenk, 2011:7). Historische Geographie wäre in diesem Sinne allerdings nicht nur jener vermeintlich ,abgehangene Traditionsbestand" der Kulturlandschaftsforschung, mit dem sie häufig gleichgesetzt wird.

\footnotetext{
${ }^{1}$ Zugleich, dies sei einschränkend angemerkt, spiegelt sich darin die unterschiedliche institutionelle Verortung und akademische Vita ihrer beiden Autoren: Während Helmut Jäger Lehrstuhlinhaber für Kulturgeographie in Würzburg war und Dissertation und Habilitation im Fach Geographie ablegte, war Klaus Fehn, eher aus der Landesgeschichte kommend, Direktor und Lehrstuhlinhaber im einem eigenständigen Seminar für Historische Geographie in Bonn, dem bis zur Überführung in das Bonner Geographische Institut 2001 einzigen in Deutschland. Vor Fehn war die Professur von dem Althistoriker Ernst Kirsten besetzt, der allerdings in der Geographie kaum rezipiert wurde. Insofern sind die Berufung Fehns und sein Engagement in der Zusammenarbeit mit der Geographie bereits als erste Annäherung an die Geographie zu werten.
}

Wie sehr sich Geographie und Historische Geographie im deutschsprachigen Diskurs bis in jüngste Zeit entfremdet haben, wird besonders deutlich, wenn man die Adaption der angelsächsischen new cultural geography in der deutschsprachigen Neuen Kulturgeographie betrachtet ${ }^{2}$. In ihrer Kritik an einer einzig auf Kulturlandschaftsforschung ausgerichteten Kulturgeographie der Sauer-Schule ähnelte sie der Kritik der Sozialgeographie. Jedoch waren es in der frühen new cultural geography sich explizit als Historische Geographen verstehende wie Denis Cosgrove, James S. Duncan, Derek Gregory und Peter Jackson, die mit neuen Konzepten und Theorien arbeiteten, aber weiterhin historisch-geographisch (und mit Bezug zur Kulturlandschaft) forschten. In einem Nachruf auf Cosgrove heißt es sogar:
„[I]n his writing and in his teaching Denis Cosgro- ve articulated what I regard as a profoundly histori- cal sense of culture and landscape. Indeed it would be no exaggeration to suggest that his work on landscape and mapping offered a broader vision of what historical geography could be, re-connecting the sub-discipline to wider currents within arts and humanities which were themselves related to new versions of what thinking historically might mean " (Driver, 2009:2).

New cultural geography ist heute im angelsächsischen Raum historical and cultural geography. In der deutschsprachigen Neuen Kulturgeographie hingegen befürchtet Andreas Dix schon auf der ersten Tagung zur Neuen Kulturgeographie 2003 eine Marginalisierung der historischen Erkenntnisperspektive und bekommt wenig später sogar den Nachweis, wenn es etwa Paul Reuber (2005:13) für ,durchaus gerechtfertigt [hält] dem bisher vorherrschenden Primat der Zeit in überspitzter Form und aus didaktischen Gründen ein zeitweiliges ,Primat des Raumes“ entgegenzusetzen“. ${ }^{3}$ Selbst dreiBig Jahre nach Kiel schien die deutschsprachige Geographie noch nicht offen für historisch-geographische Fragestellungen.

Die Entwicklung der Historischen Geographie kurz zusammenfassend betont Gebhardt, sie habe vor 1970 zum „Markenkern“ der Geographie gehört, jedoch im Kontext sozialgeographischer, handlungs- und akteursorientierter und poststrukturalistischer Ansätze massiv an Bedeutung verloren. Er resümiert: „Diese Wende hin zu stärker aktualistischen, gegenwartsbezogenen Betrachtungsweise war um

\footnotetext{
2 Ähnliches ließe sich auch über die Adaption der Radical Geography schreiben, deren Begründer David Harvey (1990) durchaus auch für eine (neue) Historische Geographie argumentiert.

${ }^{3}$ Auch ist es in Tradition des Kieler Geographentages ein deutschsprachiges Spezifikum, dass neben der historischen Perspektive auch der semiotische Zugang zu Kulturlandschaften, der sogar im Sammelband Kulturgeographie von Gebhardt et al. (2003) explizit als Arbeitsfeld der Neuen Kulturgeographie aufgelistet wurde, nicht aus der new cultural geography übernommen wurde (siehe dazu auch: Steinkrüger, 2013:14-26).
} 
1970 zweifellos überfällig, aber seitdem wurde in mancherlei Hinsicht das Kind mit dem Bade ausgeschüttet" (Gebhardt, 2011:221). Es ist jedoch fraglich, ob eine konzeptionell und theoretisch neu aufgestellte und gesellschaftlich relevante Geographie, wie sie in und nach Kiel sicherlich $\mathrm{zu}$ recht gefordert wurde, zugleich aktualistisch und geschichtsvergessen werden musste. Zudem überbetont eine solche Geschichtsschreibung, dass Großteile der deutschsprachigen Historischen Geographie weiterhin die historisch-gewachsene Kulturlandschaft ins Zentrum rückten, um eine Anschlussfähigkeit in benachbarte Fächer zu ermöglichen und sich Anwendungsfelder zu erschließen. Sie übersieht andererseits, dass mit dem von Hans-Jürgen Nitz (1993) herausgegeben Band zum Weltsystemansatz Immanuel Wallersteins oder mit Dietrich Deneckes (1992) Arbeiten zur ideologischen Aufladung von Landschaften durchaus auch alternative Impulse für mögliche theoretischkonzeptionelle Ausrichtungen gesetzt wurden, die zum Teil Fachdiskurse der Geographie antizipierten oder vorweggriffen. Auch könnten rückblickend empirischen Arbeiten etwa von Winfried Schenk (1988, 1996) zur Raumwirksamkeit des Zisterzienserordens oder zu Waldnutzungen in der Frühen Neuzeit im mittleren Deutschland durchaus in einem neuem Licht als „Dekonstruktion“ gelesen werden, wenn sie den Mythos der Zisterzienser als Gestalter von Kulturlandschaft oder des Försters als „Retter des Waldes“ aufbrechen. Letztlich bedarf es sicherlich auch eines erneuten Blicks in die Sozialgeographie nach Kiel. War sie wirklich so geschichtsblind, wie sie heute wahrgenommen wird? Waren historische Fragestellungen so sehr an den Rand gedrängt, wie es Nitz beschreibt?

Kiel ist nicht nur Mythos eines Ausbruchs aus einem vorwissenschaftlichen Verständnis von Geographie, sondern auch Ankerpunkt einer Spaltung in „Wir“ - die gegenwartsorientierte, gesellschaftlich relevante, ,wissenschaftliche“ Geographie - und „die“ - die vermeintlich stehengebliebenen, ewig gestrigen Historischen GeographInnen. In einem solchen Weltbild verbleibt Historische Geographie als ein Subalternes unter Rechtfertigungs- und Anpassungszwang. Die über die Jahre aus der Gegenwartsgeographie offen formulierte Forderung, die Historische Geographie den aktuellen Fachdiskursen anzupassen, spiegelt nicht nur hegemoniale Strukturen innerhalb der Geographie wider, sondern verleugnet die personellen Möglichkeiten und ignoriert die Eigenart historischen Arbeitens. Statt der Forderung nach Anpassung des Subalternen, böte die eigene Einlassung auf historische Tiefe eine sinnvolle Alternative. Man würde dann allerdings auch sehen, dass Quellenangebote limitierend auf die Möglichkeiten der Bearbeitung bestimmter Themen wirken können und nicht alle formulierten Wünsche methodisch einlösbar sind. Aber in solchen Quellen und Archiven könnten auch neue Forschungsfragen und Antworten $\mathrm{zu}$ finden sein, für die der Blick durch das mythische Kiel bisher verstellt ist.
Langsam entsteht eine neue Generation von Geographinnen und Geographen, die diese Spaltung nicht mehr erlebt hat, das alte Feindbild nicht mehr benötigt und durch die angelsächsische Kulturgeographie an historische Forschungsfragen herantritt. Jedoch hat diese Generation selten gehört, dass auch im deutschsprachigen Raum eine Historische Geographie existiert, denn der Mythos Kiel hatte die Geographie von seiner historischen Perspektive entfremdet.

Edited by: B. Korf

Reviewed by: one anonymous referee

\section{Literatur}

Denecke, D.: Ideology in the planned order upon the land: the example of Germany, in: Ideology and landscape in historical perspective. Essays on the meanings of some places in the past, Herausgeber: Baker, A. und Biger, G., Cambridge University Press, Cambridge, 303-329, 1992.

Driver, F.: Denis Cosgrove: Historical Geography unbound, J. Hist. Geogr., 35, 1-2, 2009.

Fehn, K.: Stand und Aufgaben der Historischen Geographie, Blätter zur deutschen Landeskunde, 127, 19-45, 1975.

Gebhardt, H.: Rezension: Schenk, Winfried: Historische Geographie, Berichte zur deutschen Landeskunde, 85, 221-222, 2011.

Gebhardt, H., Reuber, P. und Wolkersdorfer, G.: Kulturgeographie. Leitlinien und Perspektiven, in: Kulturgeographie. Aktuelle Ansätze und Entwicklungen, Herausgeber: Gebhardt, H., Reuber, P. und Wolkersdorfer, G., Spektrum, Heidelberg, 1-27, 2003.

Hard, G.: Die Geographie. Eine wissenschaftstheoretische Einführung, Walter de Gruyter, Berlin, New York, 1973.

Harvey, D.: Between Space and Time. Reflections on the Geographical Imagination, Ann. Assoc. Am. Geogr., 80, 418-434, 1990.

Jäger, H.: Historische Geographie, Georg Westermann, Braunschweig, 1969.

Meckelein, W. und Borcherdt, C.: Deutscher Geographentag Kiel, 21. Bis 26. Juli 1969: Tagungsbericht und wissenschaftliche Abhandlungen, Steiner, Wiesbaden, 1970.

Nitz, H.-J.: Historische Geographie, Siedlungsforschung, 10, 211 237, 1992.

Nitz, H.-J.: The Early Modern World-System in Geographical Perspective, Fran Steiner Verlag, Stuttgart, 1993.

Reuber, P.: Writing History - Writing Geography. Zum Verhältnis von Zeit und Raum in Geschichte und Geographie, Geogr. Z., 93, 5-16, 2005.

Schenk, W.: Mainfränkische Kulturlandschaft unter klösterlicher Herrschaft. Die Zisterzienserabtei Ebrach als raumwirksame Institution vom 16. Jh. bis 1803, Geographisches Institut der Universität Würzburg und Forschungskreis Ebrach, Würzburg und Ebrach, 1988.

Schenk, W.: Waldnutzung, Waldzustand und regionale Entwicklung in vorindustrieller Zeit im mittleren Deutschland. Historischgeographische Beiträge zur Erforschung von Kulturlandschaften in Nordhessen und Mainfranken, Franz Steiner Verlag, Stuttgart, 1996. 
Schenk, W.: Historische Geographie, WBG, Darmstadt, 2011.

Steinkrüger, J.-E.: Thematisierte Welten: Über Darstellungspraxen in Zoologischen Gärten und Vergnügungsparks, Transcript, Bielefeld, 2013. 